\title{
Simulative Analysis of the Impact of Non-Linearity in Terms of BER, Q-Factor and Jitter in Optical Soliton Transmission at $40 \mathrm{~GB} / \mathrm{S}$.
}

\author{
Manjit Singh \\ Assistant Professor \\ Deptt. Of ECE, \\ GNDU,RC, Jalandhar
}

\author{
Himali Sarangal \\ Assistant Professor \\ Deptt. Of ECE, \\ GNDU, RC, Jalandhar
}

\author{
Jyoteesh Malhotra \\ Associate Professor \\ Deptt. Of ECE, GNDU,RC,Jalandhar
}

\begin{abstract}
In this paper we have analyzes the impact of chirp factor in terms of Q-factor, BER and jitter with different dispersion parameters for optical Soliton transmission links at $40 \mathrm{~Gb} / \mathrm{s}$. The results show that BER is minimum, Q-factor is maximum and the jitter value is minimum at or near 0.7 values of chirp factor, also, the performance Soliton transmission system has optimum value. Further, it has been indicated that BER is increased, Q-factor is decreased and jitter is increased from third order of PMD to second order of PMD to first order of PMD at chirp factor of 0.7 value at which optimum transmission of soliton is done.
\end{abstract}

\section{Keywords}

Soliton, BER, Chirp-factor, Q-factor, Jitter, NRZ, GVD

\section{INTRODUCTION}

Due to ever increasing demand for higher information rate and hence the bandwidth, the suitable media for fulfilling such a requirement, optical fiber has proved to be one of best media alternative due to its high information carrying capacity. The optical fiber can offer 100's of THz bandwidth provided it is exploited practically. At present the input bit rates of $10 \mathrm{~Gb} / \mathrm{s}$ and $20 \mathrm{~Gb} / \mathrm{s}$ for a single channel are commonly used in optical communication systems and now we are using $40 \mathrm{~Gb} / \mathrm{s}$ for commercial purpose. The dispersion phenomenon is a problem for high bit rate and long haul optical communication systems. An easy solution of this problem is optical soliton's pulses that preserve the shape over long distances $[1,2]$.

The term soliton (formed from Latin solitarius - solitary) is one of the fundamental unifying ideas in modern theoretical physics and mathematics. Solitons are a special breed of optical pulses that can propagate through an optical fiber undistorted for tens of thousands of kilometers by using optical amplifiers. The key to soliton formation is the careful balance of the opposing forces of group velocity dispersion (GVD) and self-phase modulation (SPM). SPM is the frequency change caused by a phase shift induced by the pulse itself. The group velocity of a signal is function of wavelength, therefore each spectral component can be assumed to travel independently and to undergo a group delay, which ultimately results in pulse broadening. So by carefully balancing the SPM and GVD, a pulse may propagate through a fiber without any broadening in the time or frequency domains [12]

An impressive practical implementation of the soliton concept has been achieved in fiber optics, where soliton pulses are used as the information carriers to transmit digital signals over long-haul. Soliton based optical communication systems can be used over distance of several thousands of kilometers with huge information carrying capacity by using optical amplifiers. But there are number of factors which cause the degradation in the performance of optical soliton system and the one of them is Chirp factor. The chirp factor is the deviation of frequency of light waves from its central frequency and results in the broadening of optical pulses and thereby decreasing the rate of information. The value of chirp is given by:

$\delta f(t)=\left(\frac{\alpha_{e}}{4 \pi}\right)\left[\frac{d}{d t} \ln (\Delta P)+\chi \Delta P\right]$

Where $\alpha \mathrm{e}$ is line width enhancement factor, $\Delta \mathrm{P}$ is variation in optical power variation and $\chi$ is constant. The above equation can be divided into two parts: One is known as transient chirp and caused by the relaxation oscillator and second one is adiabatic chirp and it is produced by change in carrier densities because of power variation $\Delta \mathrm{p}$ in the present work the effect of chirp factor on bit error rate-value is study at the rate of $40 \mathrm{~Gb} / \mathrm{s}$ [13].

In this paper, we have analyzed the impact of Chirp factor in terms of BER, Q-factor and jitter on optical soliton transmission system with different dispersion parameters having input bit rate $40 \mathrm{~GB} / \mathrm{s}$. The BER can be estimated from equation (2), and requires Q $>6$ for the BER 10-9. This BER gives the upper limit for the signal because some degradation occurs at the receiver end.

$\mathrm{BER}=1 / 2 \operatorname{erfc}(\mathrm{Q} / \sqrt{ } 2)$

\section{SYSTEM DESCRIPTION}

Typical optical soliton transmission system consists of a transmitter, optical fiber as a channel, and receiver. The soliton communication system requires an optical source capable of producing pulses at a high repetition rate with a shape closest to the "sech" shape. The source should operate in the wavelength region near $1.55 \mu \mathrm{m}$. Receivers can feature detectors based on either PIN or APD with aperture and optional error-correction. The soliton transmission system is shown in figure 1 Is to calculate the impact of chirp factor on performance characteristics of optical communication system using optical solitons. This model is a subsystem of transmitter telescope, optical fiber and receiver telescope. The first stage in transmitter section consists of PRBS (pseudorandom bit sequence) generator. Parameter bit rate, order number of leading and trailing zeros are used in the internal PRBS generator. A different seed will be used for each bit sequence for each WDM channel. The second stage is coding/ modulation. NRZ coding is generated by the engines of NRZ 
pulse generator. In this set up we are taking NRZ format. The transmitter section consists of data source having a bit rate of $40 \mathrm{~Gb} / \mathrm{s}$, linear modulator and soliton pulse generator operating at a wavelength range of $1550 \mathrm{~nm}$, transmitted power is $70 \mathrm{mw}$ and frequency spacing $100 \mathrm{GHz}$. In this set up the length of the fiber is 70 Spans of $50 \mathrm{KM}$ each D17 type + 10,625 DCF type. We are using the in-line-erbium-doped fiber amplifier (EDFA), which amplifies the pulse through a process of stimulated emission similar to what occur in a laser [13].

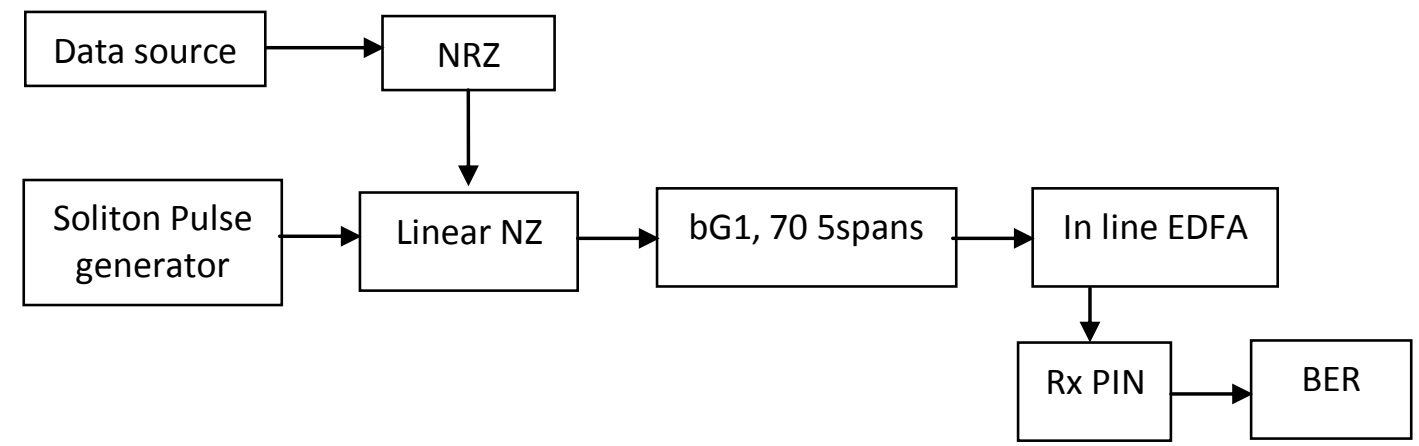

Fig.1. Design layout of soliton communication system

The receiver is a PIN type and is followed by an electrical splitter, BER detector and electrical oscilloscope. Soliton transmission system has been numerically simulated for examine the effect of chirp factor in terms of BER, Q-factor and jitter are shown in Fig.1. The simulation is carried out for three different GVD parameters as shown in table 1.

Table 1. GVD parameters

\begin{tabular}{lll}
\hline Case & Order of dispersion & Value \\
\hline Case I & Fourth & $\beta_{2}=-21.682 \mathrm{ps}^{2} / \mathrm{km}$, \\
& & $\beta_{3}=0.0141 \mathrm{ps}^{3} / \mathrm{km}$, \\
& & $\beta_{4}=-0.0068 \mathrm{ps}^{4} / \mathrm{km}$ \\
Case II & \multirow{2}{*}{ Third } & $\beta_{2}=-21.682 \mathrm{ps}^{2} / \mathrm{km}$, \\
& & $\beta_{3}=0.0141 \mathrm{ps}^{3} / \mathrm{km}$, \\
& & $\beta_{4}=-0 \mathrm{ps}^{4} / \mathrm{km}$ \\
Case III & \multirow{2}{*}{ Second } & $\beta_{2}=-21.682 \mathrm{ps}^{2} / \mathrm{km}$, \\
& & $\beta_{3}=0 \mathrm{ps}^{3} / \mathrm{km}^{4}$, \\
& & $\beta_{4}=-0 \mathrm{ps}^{4} / \mathrm{km}$ \\
\hline
\end{tabular}

\section{RESULTS AND DISCUSSION}

The right choice of the performance evaluation criteria for the characterization of optical transmission links represent one of the key issuses for an effective design of future long-haul optical systems. The evaluation criteria should provide a precise determination and separation of dominant system limitations, making them crucial for the suppression of propagation disturbances and a performance improvement. The most widely used performance evaluation are the Qfactor, BER and jitter. The chirp factor is the deviation of frequency of light waves from its central frequency and results in the broadening of optical pulses and thereby decreasing the rate of information. Optical Soliton transmission system has been numerically simulated for examine the impact of chirp factor in terms of BER, Q-factor and jitter with different order of GVD parameters is shown in Fig.1. For simulation following parameters are used: frequency of the transmitter is $1550 \mathrm{~nm}$, transmitted power is $70 \mathrm{mw}$ and frequency spacing $100 \mathrm{GHz}$. BER versus chirp, Qfactor verses chirp and jitter verses chirp plots are shown in Fig.2-4. For every simulation, the simulation parameters are same.
In case I i.e. when the order of dispersion is higher or fourth the GVD parameters are $\beta 2=-21.682 \mathrm{ps} 2 / \mathrm{km}, \beta 3=0.0141$ $\mathrm{ps} 3 / \mathrm{km}, \beta 4=-0.0068 \mathrm{ps} 4 / \mathrm{km}$.It is quite evident from the above table that as the chirp factor varies the bit error and Q-factor also fluctuates and in general deteriorates as the chirp factor varies from $0(\mathrm{BER}=2.367 * 1014)$ to $0.6(\mathrm{BER}=1.736 * 10-11)$. This is due to the fact that chirp factor (which indicates the change in frequency) in this range is not compatible with the system design as the systems normally operates optimally with chirp factor of 0.7 . The above table clearly shows the improvement in bit error rate with the chirp factor in the range of $0.7(\mathrm{BER}=4.398 * 10-15)$ to $1(\mathrm{BER}=2.858 * 10-18)$. Further the values of Q-value at chirp factor 0.7 is 18.2716 and at chirp factor 0.8 is 19.046 . Hence quality factor is improved at the value of 0.7 and jitter has minimum value at chirp factor 0.7 it is .001253 . The jitter is alter from 0.002255 at 0 chirp factor to 0.003301 at 0.6 chirp factor so minimum value of jitter is at 0.7

In case II the fourth order dispersion is compensated in that case we have taken the parameters of GVD up to third order i.e. $\beta 2=-21.682 \mathrm{ps} 2 / \mathrm{km}, \beta 3=0.0141 \mathrm{ps} 3 / \mathrm{km}, \beta 4=-0 \mathrm{ps} 4 / \mathrm{km}$. It has been observed that the BER is minimum at or near 0.7 value of chirp factor. It is deteriorates as the chirp factor varies from $0(\mathrm{BER}=9.348 * 10-14)$ to $0.6(\mathrm{BER}=2.041 * 10$ $23)$. The table 2 clearly shows the improvement in bit error rate with the chirp factor in the range of $0.7(\mathrm{BER}=3.642 * 10$ 14)to $1(B E R=1.934 * 10-17)$. Further the values of $Q$-value at chirp factor 0.7 is 18.1968 and at chirp factor 0.8 is 19.2137. Hence quality factor is improved at the value of 0.7 and jitter has minimum value at chirp factor 0.7 it is 0.001384 . The jitter is fluctuate from 0.002490 at 0 chirp factor to 0.001384 at 0.6 chirp factor so minimum value of jitter is at 0.7

In case III the fourth order and third order dispersion is compensated in that case we have taken the parameters of GVD up to second order i.e. $\beta 2=-21.682 \mathrm{ps} 2 / \mathrm{km}, \quad \beta 3=0$ ps $3 / \mathrm{km}, \beta 4=-0 \mathrm{ps} 4 / \mathrm{km}$. It has been observed that the BER is minimum at or near $0.7(\mathrm{BER}=1.467 * 10-12)$ value of chirp.

The results show that at or near chirp factor 0.7 the optimal results are obtained and quality factor is improved. The value of $\mathrm{Q}$ factor varies from 19.4814 to 18.8351 to 19.7745 to 18.4776. It has been also observed that at and near chirp factor 0.7 the optimal results are obtained the values of Qvalue at chirp factor 0.7 is 18.0144 and at chirp factor 0.8 is 18.7955. Further the observations also show that minimum 
value of jitter is at 0.7 . The jitter is fluctuate from 0.003498 at 0 chirp factor to 0.003392 at 0.6 chirp factor so all of this alteration is due to the imperfection of fiber. So the fiber behavior is improved at or above the value of chirp factor value of 0.7. Figure 2-4, shows the comparative analysis of BER, Q-factor and jitter at $40 \mathrm{~Gb} / \mathrm{s}$ with variable chirp factor with 4 th, 3rd and 2nd order of dispersion.

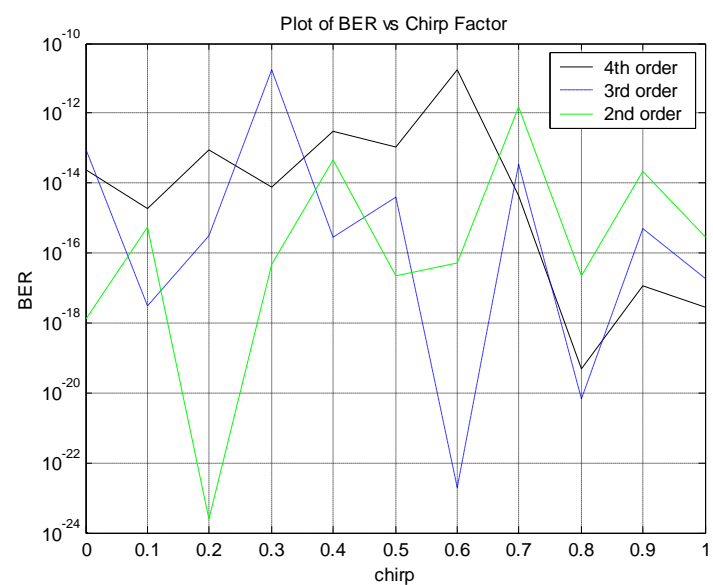

Figure 2. Plot of BER with variable chirp factor with different order of dispersion.

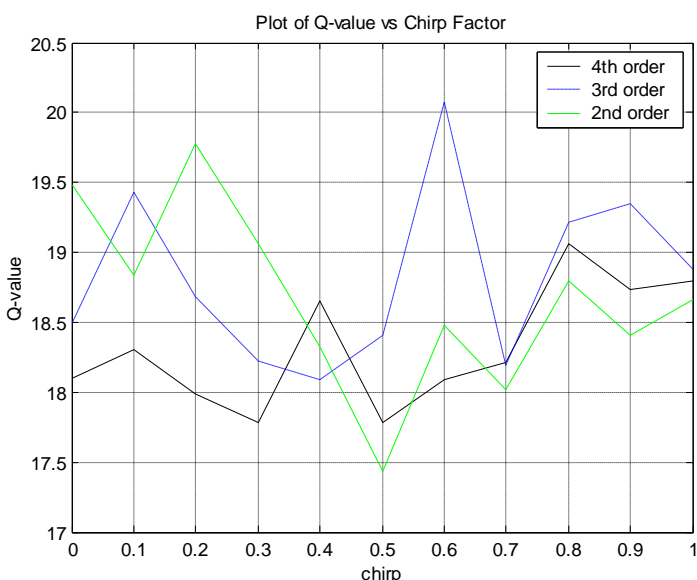

Figure 3. Plot for Q-values with variable chirp factor with different order of dispersion

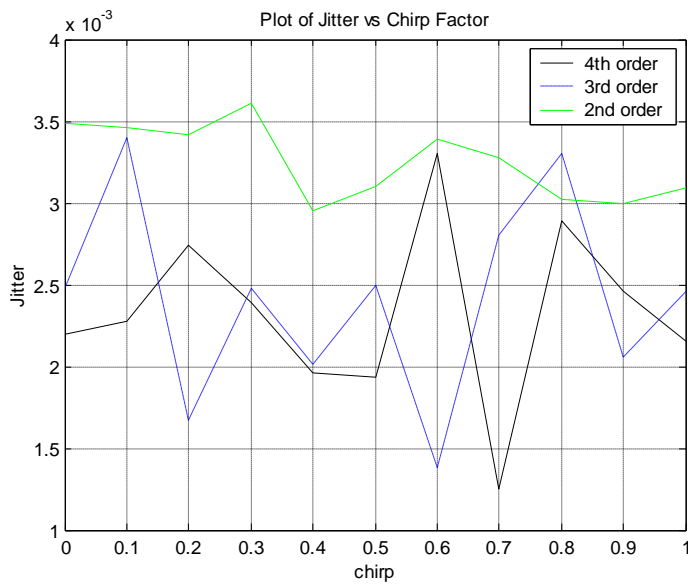

Figure 4. Plot for Jitter with variable chirp factor with different order of dispersion.

\section{CONCLUSION}

It is concluded from the all above observation that the bit error rate is minimum at or near 0.7 value of chirp factor and the performance soliton transmission system have optimum value, with $(\beta 2=-21.682 \mathrm{ps} 2 / \mathrm{km}, \beta 3=0.014 \mathrm{ps} 3 / \mathrm{km}, \beta 4=-$ $0.006 \mathrm{ps} 4 / \mathrm{km}),(\beta 2=-21.682 \mathrm{ps} 2 / \mathrm{km}, \beta 3=0.014 \mathrm{ps} 3 / \mathrm{km}, \beta 4=0$ ps $4 / \mathrm{km}),(\beta 2=-21.682 \mathrm{ps} 2 / \mathrm{km}, \beta 3=0 \mathrm{ps} 3 / \mathrm{km}, \beta 4=0 \mathrm{ps} 4 / \mathrm{km})$ respectively. From the above result it can also be concluded that in soliton transmission system the bit error rate is improved as bit error rate is increased from third order of PMD ( with $\beta 2=-21.682 \mathrm{ps} 2 / \mathrm{km}, \beta 3=0.014 \mathrm{ps} 3 / \mathrm{km}, \beta 4=-$ $0.006 \mathrm{ps} 4 / \mathrm{km}$ ) to second order of PMD ( with $\beta 2=-21.682$ $\mathrm{ps} 2 / \mathrm{km}, \beta 3=0.014 \mathrm{ps} 3 / \mathrm{km}, \beta 4=0 \mathrm{ps} 4 / \mathrm{km})$ to first order of PMD ( with $(\beta 2=-21.682$ ps $2 / \mathrm{km}, \beta 3=0$ ps $3 / \mathrm{km}, \quad \beta 4=0$ ps $4 / \mathrm{km}$ ) at chirp factor of value 0.7 at which optimum transmission of solution is done. Similarly the value of quality factor is decreased from third order of PMD ( with $\beta 2=-21.682 \mathrm{ps} 2 / \mathrm{km}, \beta 3=0.014 \mathrm{ps} 3 / \mathrm{km}, \beta 4=-0.006 \mathrm{ps} 4 / \mathrm{km}$ ) to second order of PMD ( with $\beta 2=-21.682 \mathrm{ps} 2 / \mathrm{km}, \beta 3=0.014$ ps $3 / \mathrm{km}, \beta 4=0 \mathrm{ps} 4 / \mathrm{km})$ to first order of PMD ( with $(\beta 2=-$ $21.682 \mathrm{ps} 2 / \mathrm{km}, \beta 3=0 \mathrm{ps} 3 / \mathrm{km}, \beta 4=0 \mathrm{ps} 4 / \mathrm{km}$ ) at chirp factor of value 0.7 at which optimum transmission of soliton is done . And the value of jitter is increased from third order of PMD ( with $\beta 2=-21.682 \mathrm{ps} 2 / \mathrm{km}, \quad \beta 3=0.014 \mathrm{ps} 3 / \mathrm{km}, \quad \beta 4=-0.006$ $\mathrm{ps} 4 / \mathrm{km}$ ) to second order of PMD ( with $\beta 2=-21.682 \mathrm{ps} 2 / \mathrm{km}$, $\beta 3=0.014 \mathrm{ps} 3 / \mathrm{km}, \beta 4=0 \mathrm{ps} 4 / \mathrm{km}$ ) to first order of PMD ( with $(\beta 2=-21.682 \mathrm{ps} 2 / \mathrm{km}, \beta 3=0 \mathrm{ps} 3 / \mathrm{km}, \beta 4=0 \mathrm{ps} 4 / \mathrm{km})$ at chirp factor of value 0.7 at which optimum transmission of soliton is done.

\section{REFERENCES}

[1] Andrekson, Peter A. (1999) "40 Gbt/s soliton transmission on installed fiber lines" Department of microelectronics, photonics Laboratory Chalmer university of technology.

[2] Holzlöhner,Ronald et.al (2002) “ Experimental and Theoretical Characterization of a 40-Gb/s Long-Haul Single-Channe Transmission System" Journal of lightwave technology, vol. 20, no 7, pp. 1124-1131

A. Eric Swanson (2001) "100- GHz Soliton Pulse Train Generation using Soliton compression of Two Phase side bands from a single DFB Laser." IEEE photo technology letters, vol.6, no. 10, pp. 1194-1196

[3] Arahira S. et. al(2001) "Optical short pulse generation at High repetition rate over $80 \mathrm{ghz}$ from a monolithic passively Mode locked dbr laser diode". Electronics letters vol 29, no. 11 pp. page 1013-101.

[4] Chongjin Xie, et. al.(2001) "Polarization-Mode Dispersion-Induced Outages in Soliton Transmission Systems" IEEE Photonics technology letters, vol. 13, no. 10, pp. 1079-81.

[5] J. Poirrier, F. Buchali, and H. B"ulow (2001) "Electronic WDM crosstalk cancellation," in Proc. ECOC'01, vol. 1, pp. 94-94.

[6] Knox, F.M. et.al (1995) "Low jitter long distance pulse transmission near net fiber dispersion zero wavelength" Electronics letters, vol. 31 no 17 1466-67.

[7] Lakoba T. I. et.al (2000) "Optimization of the AverageDispersion Range for Long-Haul Dispersion-Managed Soliton Systems" Journal of lightwave technology, vol. 18 , no 11 , pp. $1504-13$. 
[8] Mårtensson, Jonas and Berntson, Anders et.al (2001) "Dispersion-Managed Solitons for 160-Gb/s Data Transmission" IEEE Photonics technology letters, vol. 13, no 7, pp. 666-68.

[9] Matsumoto M. (2001) "Suppression of collision-induced timing jitter in WDM RZ-pulse transmission by adjustment of decision timing," in OFC'00 Tech. Digest, Anaheim, CA, vol. 3, pp. WM3 1-3.

[10] Morita, Itsuro et.al (1999) "Long-Haul Soliton WDM Transmission with Periodic Dispersion Compensation and Dispersion Slope Compensation" Journal of lightwave technology, vol. 17, no 1, pp. 80-85
[11] Himali Sarangal et.al (2008) "Communication System Based on Soliton" in proceedings of National Conference on Optical and Wireless Communication, NCOW'2008, DAVIET, Jalandhar, Nov 27-28, 2012 pp. 267-270.

[12] Himali Sarangal and Manjit Singh (2012) "Impact of Non-linearity in terms of BER in Optical Soliton Transmission" in proceedings of International Conference on Systemics, Cybernetics and Informatics ICSCI-2012, Hyderabad, Andhra Pradesh, Feb 1518,2012 . 